\title{
Sugli indici di attività dei filamenti solari
}

\author{
G. Forti $\left({ }^{*}\right)-$ G. GodoLI $\left({ }^{*}\right)-$ G. NocI $\left({ }^{*}\right)$
}

Ricevuto il 22 Gennaio 1965

Riassunto. - Dopo aver brevemente esaminato gli indici utilizzati per la descrizione dell'attività dei filamenti solari, viene esposto ed applicato un metodo statistico che permette di individuare gli indiei migliori.

Summary. - The indices used for the description of the solar filaments activity are briefly examined. A statistical method for choosing the better indices is explained and applied.

\section{1. - INTRODUZIONE.}

Il problema dell'analisi degli indici di attività solare ha due aspetti:

a) si possono ricercare quali sono quei fenomeni solari che presentano una maggiore correlazione con un determinato fenomeno geofisico;

b) si possono ricercare quelle grandezze che meglio descrivono un determinato fenomeno solare.

Ovviamente la seconda ricerca, di carattere strettamente metrologico, è propedeutica alla prima.

Nel presente lavoro ei siamo proposti di analizzare gli indici utilizzati per la descrizione dell'attività dei filamenti solari.

Per una accurata descrizione morfologica e fisica del fenomeno delle protuberanze e dei filamenti solari rimandiamo ad altra sede (1). Qui ci preme ricordare soltanto che i filamenti oscuri, osservati sul disco in radiazione monocromatica, altro non sono che protuberanze generalmente quiescenti. Si possono occasionalmente osservare sul disco come filamenti anche protuberanze attive ma in questo caso i filamenti sono poco estesi e molto variabili.

(*) Osservatorio Astrofisico di Arcetri - Firenze. I Sezione del Centro di Astrofisica del C.N.R. 


\section{Materiale di osservazione.}

Per la descrizione dell'attività dei filamenti vengono utilizzati i seguenti indici:

\section{a) Numero giornaliero di fenomeni $N$.}

Il numero di fenomeni visibile giornalmente sul disco è senza dubbio l'indice più immediato. Questo indice può essere ricavato direttamente dal materiale di osservazione originale.

Medie semestrali del numero giornaliero di fenomeni, secondo le osservazioni di Kodaikanal, sono state pubblicate dall'Osservatorio di Kodaikanal dal 1912 con le sole lacune dell'anno 1914 e del primo semestre del 1935. Dal 1924 al 1937 e dal 1941 in poi sono state pubblicate dall'osservatorio di Kodaikanal anche le medie semestrali del numero giornaliero dei fenomeni secondo le osservazioni di Kodaikanal, integrate da quelle di altri osservatori collaboranti ( $\left.{ }^{2}\right)$.

Le medie annuali di questo indice, secondo le osservazioni di Kodai kanal e secondo le osservazioni di Kodaikanal integrate da quelle di altri osservatori collaboranti, sono riportate nella Tabella I. Data la sufficiente uniformità della distribuzione delle osservazioni durante l'anno, le medie annuali sono state dedotte mediante quelle semestrali.

\section{b) Numero di fenomeni per rotazione $\mathrm{Nr}$.}

Il numero di fenomeni presenti sul Sole durante una rotazione sinodica equivale, statisticamente, alla metà della media rotazionale del numero giornaliero di fenomeni, dato che la durata dei filamenti non varia molto, in media, da un oggetto all'altro.

Il numero di fenomeni per rotazione è facilmente ricavabile dai cataloghi di Meudon per il periodo Marzo 1919-Dicembre 1963 ( $^{3}$ ).

Le medie annuali del numero di fenomeni per rotazione sono riportate nella terza colonna della Tabella I. Le medie annuali sono state eseguite attribuendo ad ogni anno le rotazioni che in esso hanno avuto inizio. La media relativa al 1919, sebbene sia stata eseguita a partire dalla rotazione iniziatasi il 15 Marzo, non dovrebbe essere troppo diversa dalla media completa: nel periodo 1918-1920 l'attività dei filamenti si è infatti mantenuta abbastanza costante. 
Tabella I. - Medie anyuali del numero giornaliero di filamenti $N$ SECONDO LE OSSERVAZIONI DI KODAIKANAL $(K)$ E SECONDO LE OSSERVA. ZIONI DI KODAIKANAL INTEGRATE DA QUELLE DI ALTRI OSSERVATORI COLLABORANTI $(C)$.

Medie anNUALI DEL NUMERo di filamenti PER Rotazione $N_{r}$

\begin{tabular}{|c|c|c|c|c|c|c|c|}
\hline \multirow{2}{*}{ Anno } & \multicolumn{2}{|c|}{$N$} & \multirow{2}{*}{$N_{r}$} & \multirow{2}{*}{ Anno } & \multicolumn{2}{|c|}{$N$} & \multirow{2}{*}{$N_{r}$} \\
\hline & $K$ & $C$ & & & $K$ & $C$ & \\
\hline 1912 & 1.53 & & & 1938 & 51.47 & & 81.5 \\
\hline 1913 & 0.69 & & & 1939 & 49.26 & & 67.5 \\
\hline 1914 & - & & & 1940 & 41.68 & & 56.9 \\
\hline 1915 & 8.95 & & & 1941 & 27.47 & 28.27 & 42.9 \\
\hline 1916 & 11.78 & & & 1942 & 23.68 & 24.76 & 35.1 \\
\hline 1917 & 21.23 & & & 1943 & 15.26 & 17.24 & 21.0 \\
\hline 1918 & 24.15 & & & 1944 & 10.12 & 12.85 & 17.7 \\
\hline 1919 & 20.10 & & 52.3 & 1945 & 18.68 & 19.54 & 32.7 \\
\hline 1920 & 28.35 & & 45.6 & 1946 & 32.84 & 33.32 & 50.1 \\
\hline 1921 & 28.25 & & 37.6 & 1947 & 38.17 & 39.96 & 55.2 \\
\hline 1922 & 15.00 & & 28.7 & 1948 & 30.52 & 31.62 & 66.7 \\
\hline 1923 & 8.95 & & 20.2 & 1949 & 35.73 & 34.76 & 68.0 \\
\hline 1924 & 15.00 & 14.40 & 28.7 & 1950 & 26.28 & 28.31 & 50.7 \\
\hline 1925 & 20.15 & 19.55 & 41.6 & 1951 & 26.02 & 26.29 & 38.3 \\
\hline 1926 & 34.15 & 33.90 & 43.1 & 1952 & 18.70 & 19.24 & 29.0 \\
\hline 1927 & 33.90 & 33.90 & 44.1 & 1953 & 11.66 & 11.64 & 23.3 \\
\hline 1928 & 28.29 & 30.15 & 41.1 & 1954 & 5.32 & 5.80 & 13.8 \\
\hline 1929 & 25.42 & 25.40 & 38.2 & 1955 & 11.86 & 13.28 & 30.9 \\
\hline 1930 & 21.05 & 21.10 & 37.8 & 1956 & 30.26 & 29.81 & 57.2 \\
\hline 1931 & 14.56 & 14.68 & 32.2 & 1957 & 28.45 & 27.60 & 67.0 \\
\hline 1932 & 8.07 & 8.56 & 22.0 & 1958 & 29.44 & 29.35 & 76.3 \\
\hline 1933 & 6.96 & 7.34 & 18.8 & 1959 & & & 67.1 \\
\hline 1934 & 10.35 & 10.38 & 20.4 & 1960 & & & 59.3 \\
\hline 1935 & - & 23.53 & 33.6 & 1961 & & & 41.3 \\
\hline 1936 & 46.64 & 47.76 & 52.6 & 1962 & & & 31.4 \\
\hline 1937 & 54.60 & 55.46 & 74.2 & 1963 & & & 22.2 \\
\hline
\end{tabular}


c) Numero caratteristico giornaliero N.C.

Il numero caratteristico giornaliero costituisce una stima, in una scala da 0 a 5 , dell'attività giornaliera dei filamenti: 0 significa attività minima; 5 attività massima. Per diminuire la soggettività delle stime, sono stati distribuiti dagli osservatori di Monte Wilson e Meudon degli spettroeliogrammi campione.

I numeri caratteristici per i filamenti, introdotti per convenzione internazionale, sono stati pubblicati nel "Bulletin for character figures of solar phenomena" (successivamente chiamato Quarterly bulletin on solar activity) per il periodo 1917-1944: fino al 1941 sono stati pubblicati i valori giornalieri stimati nei singoli osservatori; le medie giornaliere di questi valori; le medie mensili per i singoli e per tutti gli osservatori; dal 1942 al 1944 sono state invece pubblicate soltanto le medie giornaliere e mensili per tutti gli osservatori. In tre riassunti sono date le medie annuali dei numeri caratteristici per tutti gli osservatori ( ${ }^{(4)}$.

Per il periodo 1932-1949 sono state pubblicate in questa sede $\left(^{5}\right)$ le medie mensili ed annuali dei numeri caratteristici stimati ad Arcetri. Dal 1950 al 1960 sono stati pubblicati sempre in questa sede $\left(^{8}\right)$ anche $i$ valori giornalieri. Dal 1961 i valori giornalieri dei numeri caratteristici dei filamenti stimati ad Arcetri sono stati pubblicati nel bollettino delle osservazioni eseguite all'Osservatorio Astrofisico di Arcetri (?).

È stato dimostrato (5) che i numeri caratteristici dei filamenti non sono proporzionali né all'area coperta giornalmente dai fenomeni, né al logaritmo dell'area. E possibile ottenere invece una serie di numeri proporzionali all'area coperta dai fenomeni qualora si alteri la scala dei numeri caratteristici come è indicato nel seguente schema:

\begin{tabular}{|c|c|}
\hline $\begin{array}{c}\text { Numero } \\
\text { caratteristico } \\
N . O .\end{array}$ & $\begin{array}{c}\text { Numero } \\
\text { caratteristico } \\
\text { alterato }(N . O .)_{a}\end{array}$ \\
\hline 0 & 0 \\
1 & 0.5 \\
2 & 1 \\
3 & 2 \\
4 & 3 \\
5 & 4 \\
\hline
\end{tabular}


Tabella II - Medie anndali del numero caratteristico giornaliero N.C. E del numero Caratteristico giornaliero alterato (N.C.) a Dei FilaMenti SECONDO IL QUARTERLy Bulletin $(Q)$ E SECONDO ARCETRI $(A)$.

\begin{tabular}{|c|c|c|c|c|c|c|c|c|c|}
\hline \multirow{2}{*}{$\begin{array}{l}\text { 염 } \\
\text { 련 }\end{array}$} & \multicolumn{2}{|c|}{$Q$} & \multicolumn{2}{|c|}{$A$} & \multirow{2}{*}{ 沗 } & \multicolumn{2}{|c|}{$Q$} & \multicolumn{2}{|c|}{ A } \\
\hline & N.C. & $(\text { N.C. })_{a} \mid$ & N.O. & $(N . O .)_{e}$ & & N.O. & $\left(N . C_{.}\right)_{a}$ & N.O. & $(\text { N.C. })_{a}$ \\
\hline 1917 & 2.43 & 1.43 & & & 1941 & 1.70 & 0.85 & 1.60 & 0.80 \\
\hline 1918 & 2.51 & 1.51 & & & 1942 & 1.40 & 0.70 & 0.90 & 0.45 \\
\hline 1919 & 2.11 & 1.11 & & & 1943 & 1.00 & 0.50 & 0.90 & 0.45 \\
\hline 1920 & 1.80 & 0.90 & & & 1944 & 0.90 & 0.45 & 0.70 & 0.35 \\
\hline 1921 & 1.38 & 0.69 & & & 1945 & & & 1.30 & 0.65 \\
\hline 1922 & 0.78 & 0.39 & & & 1946 & & & 2.00 & 1.00 \\
\hline 1923 & 0.43 & 0.22 & & & 1947 & & & 2.70 & 1.70 \\
\hline 1924 & 0.84 & 0.42 & & & 1948 & & & 2.30 & 1.30 \\
\hline 1925 & 1.15 & 0.58 & & & 1949 & & & 2.50 & 1.50 \\
\hline 1926 & 2.38 & 1.19 & & & 1950 & & & 2.26 & 1.26 \\
\hline 1927 & 2.00 & 1.00 & & & 1951 & & & 1.11 & 0.56 \\
\hline 1928 & 2.64 & 1.64 & & & 1952 & & & 0.81 & 0.40 \\
\hline 1929 & 2.55 & 1.55 & & & 1953 & & & 0.35 & 0.18 \\
\hline 1930 & 1.92 & 0.96 & & & 1954 & & & 0.29 & 0.14 \\
\hline 1931 & 1.23 & 0.62 & & & 1955 & & & 1.50 & 0.75 \\
\hline 1932 & 0.51 & 0.26 & 0.30 & 0.15 & 1965 & & & 2.87 & 1.87 \\
\hline 1933 & 0.35 & 0.18 & 0.20 & 0.10 & 1957 & & & 2.59 & 1.59 \\
\hline 1934 & 0.61 & 0.30 & 0.40 & 0.20 & 1958 & & & 2.23 & 1.23 \\
\hline 1935 & 1.37 & 0.68 & 0.90 & 0.45 & 1959 & & & 2.15 & 1.15 \\
\hline 1936 & 2.61 & 1.61 & 3.10 & 2.10 & 1960 & & & 1.89 & 0.94 \\
\hline 1937 & 2.98 & 1.98 & 2.80 & 1.80 & 1961 & & & 1.52 & 0.76 \\
\hline 1938 & 2.96 & 1.96 & 3.20 & 2.20 & 1962 & & & 0.89 & 0.44 \\
\hline 1939 & 2.60 & 1.60 & 2.90 & 1.90 & 1963 & & & 0.85 & 0.42 \\
\hline 1940 & 2.50 & 1.50 & 2.40 & 1.40 & & & & & \\
\hline
\end{tabular}


Le medie annuali del numero caratteristico giornaliero e del numero caratteristico giornaliero alterato, secondo il Quarterly Bulletin e secondo Arcetri, sono riportate nella Tabella II.

d) Indice rotazionale di attività I secondo Meudon.

All'osservatorio di Meudon viene rilevato dalle carte solari un indice rotazionale di attività, separatamente per le diverse zone eliografiche di $5^{\circ}$ di ampiezza (3). Di ciascun filamento viene determinata la lunghezza compresa fra i paralleli che delimitano la zona considerata. La lunghezza

Tabella III - Media anNuale Dell'indice Rotazionale Di attività $I$ DEI FILAMENTI.

\begin{tabular}{|c|c||c|c||c|c|}
\hline Anno & \multicolumn{1}{|c||}{ I } & Anno & I & Anno & I \\
\cline { 2 - 5 } 1919 & 1244 & 1932 & 321 & 1945 & 895 \\
\cline { 2 - 5 } 1920 & 1016 & 1933 & 260 & 1946 & 1449 \\
1921 & 809 & 1934 & 353 & 1947 & 1729 \\
1922 & 624 & 1935 & 750 & 1948 & 1634 \\
1923 & 387 & 1936 & 1424 & 1949 & 1557 \\
1924 & 548 & 1937 & 1735 & 1950 & 1243 \\
1925 & 858 & 1938 & 1969 & 1951 & 858 \\
1926 & 1278 & 1939 & 1494 & 1952 & 568 \\
1927 & 1057 & 1940 & 1029 & 1953 & 448 \\
1928 & 1099 & 1941 & 803 & 1954 & 272 \\
1929 & 1151 & 1942 & 627 & 1955 & 763 \\
1930 & 930 & 1943 & 397 & 1956 & 1555 \\
1931 & 560 & 1944 & 377 & 1957 & 1592 \\
\hline
\end{tabular}

viene poi noltiplicata per 1,2,3, rispettivamente a seconda che sulle carte il filamento sia lasciato in bianco, trattegriato o tinto in nero. (Ricordiamo che ciò equivale a dare peso 1, 2, 3 rispettivamente a quei fenomeni la cui durata sia stata inferiore ad un terzo della durata della visibilità della regione interessata, oppure compresa fra un terzo e due 
terzi, oppure superiore a due terzi). Il valore pesato della lunghezza viene poi moltiplicato per il coseno della latitudine media della zona considerata al fine di tener conto della deformazione delle lunghezze sulle carte. Il numero cosí ottenuto viene poi sommato ai numeri ottenuti per gli altri filamenti nella zona considerata.

Sommando opportunamente gli indici relativi alle varie zone si ottengono indici rotazionali per i filamenti polari o per quelli equatoriali; per un emisfero o per tutto il globo.

Questi indici sono ricavabili dai cataloghi di Mendon per il periodo 15 Marzo 1919 - 30 Giugno 1957.

Le medie annuali dell'indice rotazionale di attività $I$ per tutto il globo sono riportate nella Tabella III. Come abbiamo già osservato la media relativa al 1919, sebbene sia stata eseguita a partire dalla rotazione iniziatasi il 15 Marzo, non dovrebbe essere troppo diversa da quella completa, invece la media relativa al $\mathbf{1 9 5 7}$, eseguita sulla base delle rotazioni iniziatesi nel primo semestre dell'anno, potrebbe anche essere molto diversa dalla media completa: per questo essa è riportata fra parentesi.

\section{e) Area coperta giomalmente.}

L'indice, la cui determinazione richiede senza dubbio il massimo impegno, è l'area coperta giornalmente dai fenomeni.

E noto che l'area coperta da un fenomeno solare, misurata sul disco, viene chiamata «area proiettata ». Moltiplieando l'area proiettata per la secante dell'angolo centro disco-centro Sole-baricentro del fenomeno, si ottiene l'uarea corretta ». Evidentemente, l'" area corretta " coincide con l'“ area vera » del fenomeno solo se questo giace sulla superficie solare. Per questo, quando è stata scoperta la costituzione spaziale dei filamenti solari, ci si è limitati a determinarne l'area proiettata.

Medie semestrali dell'area corretta coperta giomalmente dai filamenti secondo le osservazioni di Kodaikanal sono state pubblicate dall'Osservatorio di Kodaikanal dal 1912 al 1940 con le sole lacune dell'anno 1914 e del primo semestre del 1935. Dal 1924 al 1938 sono state pubblicate dall'Osservatorio di Kodaikanal anche le medie semestrali secondo le osservazioni di Kodaikanal, integrate da quelle di altri osservatori collaboranti (2).

Dal 1929, l'Osservatorio di Kodaikanal ha pubblicato anche le medie semestrali dell'area proiettata coperta giornalmente dai filamenti secondo le osservazioni di Kodaikanal integrate da quelle di alliri osservatori collaboranti $\left({ }^{2}\right)$. 
Tabella IV - Medie anNuali dell'area proiettata $(p)$ E Corretta $(c)$ COPERTa giornalmente daI FILAMENTI SECONDO LE OSSERVAzIONi di KODaIKaNAL $(\boldsymbol{K})$; SECONDO LE OSSERVAZIONI DI KODAIKaNaL INTEGRaTE DA QUELLE DI ALTRI OSSERVATORI COLlaboranti $(C)$ E SECONDO LE osservazioni di Arcetri ( $A$ ). Le aree sono espresse in MilioneSIMI DELL'EMISFERO.

\begin{tabular}{|c|c|c|c|c|c|c|c|c|c|c|c|}
\hline \multirow{2}{*}{ 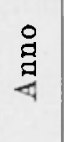 } & \multicolumn{2}{|c|}{$K$} & \multicolumn{2}{|c|}{$C$} & \multirow{2}{*}{$\frac{A}{p}$} & \multirow{2}{*}{ 号 } & \multicolumn{2}{|c|}{$\Pi$} & \multicolumn{2}{|c|}{$C$} & \multirow{2}{*}{$\begin{array}{c}A \\
p\end{array}$} \\
\hline & $p$ & $c$ & $p$ & $c$ & & & $p$ & $c$ & $p$ & $c$ & \\
\hline 1912 & & 386 & & & & 1936 & & 8928 & 4850 & 9064 & 3550 \\
\hline 1913 & & 94 & & & & 1937 & & 9320 & 5114 & 9420 & 4550 \\
\hline 1914 & & - & & & & 1938 & & 10916 & 6037 & 10562 & 4200 \\
\hline 1915 & & 2542 & & & & 1939 & & 9716 & 5414 & & 4200 \\
\hline 1916 & & 1844 & & & & 1940 & & 7795 & 4432 & & 2950 \\
\hline 1917 & & 3246 & & & & 1941 & 3146 & & 3024 & & 1200 \\
\hline 1918 & & 3610 & & & & 1942 & 2735 & & 2990 & & 900 \\
\hline 1919 & & 3496 & & & & 1943 & 1686 & & 1796 & & 650 \\
\hline 1920 & & 4816 & & & & 1944 & 1032 & & 1340 & & 200 \\
\hline 1921 & & 3584 & & & , & 1945 & 2122 & & 2184 & & 550 \\
\hline 1922 & & 1758 & & & & 1946 & 4372 & & 4358 & & 3200 \\
\hline 1923 & & 1044 & & & & 1947 & 4605 & & 4672 & & 3750 \\
\hline 1924 & & 2009 & & 1920 & & 1948 & 3384 & & 3354 & & 3250 \\
\hline 1925 & & 2532 & & 2532 & & 1949 & 4531 & & 4304 & & 4050 \\
\hline 1926 & & 4734 & & 4672 & & 1950 & 3143 & & 3336 & & \\
\hline 1927 & & 3731 & & 3885 & & 1951 & 2808 & & 2917 & & \\
\hline 1928 & & 4132 & & 4261 & & 1952 & 1812 & & 1935 & & \\
\hline 1929 & & 4238 & 2542 & 4336 & & 1953 & 1123 & & 1142 & & \\
\hline 1930 & & 3350 & 1998 & 3396 & & 1954 & 559 & & 609 & & \\
\hline 1931 & & 2138 & 1226 & 2184 & & 1955 & 1866 & & 1987 & & \\
\hline 1932 & & 1256 & 682 & 1301 & 450 & 1956 & 4906 & & 4820 & & \\
\hline 1933 & & 1058 & 610 & 1080 & 450 & 1957 & 5018 & & 4924 & & \\
\hline 1934 & & 2036 & 1037 & 2062 & 250 & 1958 & 4854 & & 4802 & & \\
\hline 1935 & & - & 2293 & 4737 & 900 & & & & & & \\
\hline
\end{tabular}


Medie mensili ed annuali di questo indice secondo le osservazioni di Arcetri, sono state pubblicate dall'Osservatorio di Arcetri per il periodo $1932-1949\left(^{8}\right)$.

Le medie annuali delle aree coperte giornalmente secorido le osservazioni disponibili di Kodaikanal; di Kodaikanal integrate da quelle di altri osservatori collaboranti; di Arcetri, sono riportate nella Tabella IV. Data la sufficiente uniformità della distribuzione delle osservazioni durante l'anno, le medie annuali per Kodaikanal e per Kodaikanal ed osservatori collaboranti sono state dedotte mediando quelle semestrali.

\section{3. - ANALISI DEGLI INDICI DISPONIBII.}

Per individuare quali siano, fra quelli disponibili, gli indici più adatti per la descrizione dell'attività dei filamenti solari, abbiamo preso in considerazione il periodo $1919-1956$ per cui sono disponibili tutti gli indici.

I dati analizzati sono stati riportati nella Tabella V.

Per il numero giornaliero di filamenti $N$ sono state considerate le medie annuali secondo le osservazioni di Kodaikanal per il periodo 19191923 e le modie annuali secondo le osservazioni di Kodaikanal integrate da quelle di altri osservatori collaboranti per il periodo 1924-1956.

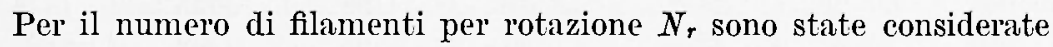
le medie annuali secondo Meudon. Per quanto abbiamo detto al n. $2 \mathrm{~b}$ è stata considerata anche la media relativa al 1919 .

Per il numero caratteristico giornaliero $N$.C. e per il numero caratteristico giornaliero alterato $(N . C .)_{a}$ sono state considerate le medie annuali secondo il Quarterly Bulletin per il periodo 1919-1944 e le medie annuali secondo Arcetri per il periodo 1945-1956.

Per l'indice di attività $I$ sono state considerate le medie annuali secondo Meudon. Per quanto abbiamo detto al $n$. $2 d$ è stata considerata la media relativa al 1919 ma non quella relativa al 1957.

Per l'area coperta giornalmente sono state considerate le medie annuali delle aree proiettate secondo le osservazioni di Kodaikanal integrate da quelle di altri osservatori per il periodo 1919-1956. Per i periodi 1919-1923 e 1921-1928 le medie considerate sono state ottenute dalle medie annuali delle aree corrette rispettivamente secondo le osservazioni di Kodaikanal e secondo le osservazioni di Kokaikanal integrate da quelle di altri osservatori collaboranti, applicando il fattore di ridnzione 0.553. Questo fattore è stato ricavato mediando per il periodo 
Tabella V. - INDici analizzati deI FILAMENTI.

\begin{tabular}{|c|c|c|c|c|c|c|}
\hline Anno & $N$ & $N_{r}$ & $N . C$. & $(N . C .)_{a}$ & $I$ & $A$ \\
\hline 1919 & 20.10 & 52.3 & 2.11 & 1.11 & 1244 & 1933 \\
\hline 1920 & 28.35 & 45.6 & 1.80 & 0.90 & 1016 & 2664 \\
\hline 1921 & 28.25 & 37.6 & 1.38 & 0.69 & 809 & 1982 \\
\hline 1922 & 15.00 & 28.7 & 0.78 & 0.39 & 624 & 972 \\
\hline 1923 & 8.95 & 20.2 & 0.43 & 0.22 & 387 & 578 \\
\hline 1924 & $14.4 \mathrm{C}$ & 28.7 & 0.84 & 0.42 & 548 & 1062 \\
\hline 1925 & 19.55 & 41.6 & 1.15 & 0.58 & 858 & 1395 \\
\hline 1926 & 33.90 & 43.1 & 2.38 & 1.19 & 1278 & 2584 \\
\hline 1927 & 33.90 & 44.1 & 2.00 & 1.00 & 1057 & 2148 \\
\hline 1928 & 30.15 & 41.1 & 2.64 & 1.64 & 1099 & 2356 \\
\hline 1929 & 25.40 & 38.2 & 2.55 & 1.55 & 1151 & 2542 \\
\hline 1930 & 21.10 & 37.8 & 1.92 & 0.96 & 930 & 1998 \\
\hline 1931 & 14.68 & 32.2 & 1.23 & 0.62 & 560 & 1226 \\
\hline 1932 & 8.56 & 22.0 & 0.51 & 0.26 & 321 & 682 \\
\hline 1933 & 7.34 & 18.8 & 0.35 & 0.18 & 260 & 610 \\
\hline 1934 & 10.38 & 20.4 & 0.61 & 0.30 & 353 & 1037 \\
\hline 1935 & 23.53 & 33.6 & 1.37 & 0.68 & 750 & 2293 \\
\hline 1936 & 47.76 & 52.6 & 2.61 & 1.61 & 1424 & 4850 \\
\hline 1937 & 55.46 & 74.2 & 2.98 & 1.98 & 1735 & 5114 \\
\hline 1938 & 53.98 & 81.5 & 2.96 & 1.96 & 1969 & 6037 \\
\hline 1939 & 49.26 & 67.5 & 2.60 & 1.60 & 1494 & 5414 \\
\hline 1940 & 41.68 & 56.9 & 2.50 & 1.50 & 1029 & 4432 \\
\hline 1941 & 28.27 & 42.9 & 1.70 & 0.85 & 803 & 3024 \\
\hline 1942 & 24.76 & 35.1 & 1.40 & 0.70 & 627 & 2990 \\
\hline 1943 & 17.24 & 21.0 & 1.00 & 0.50 & 397 & 1796 \\
\hline 1944 & 12.85 & 17.7 & 0.90 & 0.45 & 377 & 1340 \\
\hline 1945 & 19.54 & 32.7 & 1.30 & 0.65 & 895 & 2184 \\
\hline 1946 & 33.32 & 50.1 & 2.00 & 1.00 & 1449 & 4358 \\
\hline 1947 & 39.96 & 55.2 & 2.70 & 1.70 & 1729 & 4672 \\
\hline 1948 & 31.62 & 66.7 & 2.30 & 1.30 & 1634 & 3354 \\
\hline 1949 & 34.76 & 68.0 & 2.50 & 1.50 & 1557 & 4304 \\
\hline 1950 & 28.31 & 50.7 & 2.26 & 1.26 & 1243 & 3336 \\
\hline 1951 & 26.29 & 38.3 & 1.11 & 0.56 & 858 & 2917 \\
\hline 1952 & 19.24 & 29.0 & 0.81 & 0.40 & 568 & 1935 \\
\hline 1953 & 11.64 & 23.3 & 0.35 & 0.18 & 448 & 1142 \\
\hline 1954 & 5.80 & 13.8 & 0.29 & 0.14 & 272 & 609 \\
\hline 1955 & 13.28 & 30.9 & 1.50 & 0.75 & 763 & 1987 \\
\hline 1956 & 29.81 & 57.2 & 2.87 & 1.87 & 1555 & 4820 \\
\hline
\end{tabular}


1929-1938 i rapporti fra le medie annuali delle aree proiettate e di quelle corrette secondo le osservazioni di Kodaikanal integrate da quelle di altri osservatori.

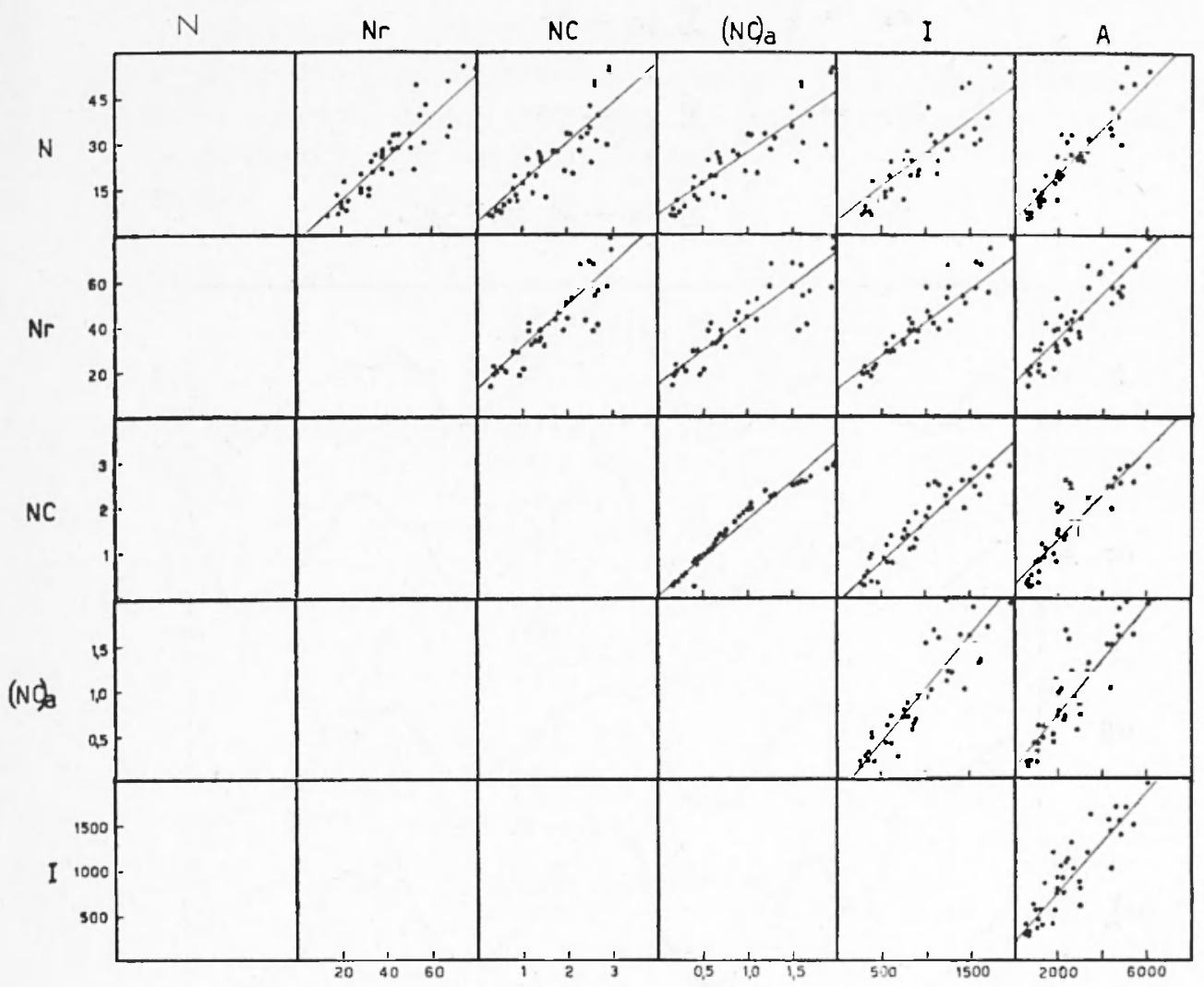

Fig. 1 - Confronto tra gli indiei analizzati.

Nei grafici di Figr. 1 gli indici analizzati sono confrontati a due a due per il periodo 1919-1956. Nel grafico di Fig. 2 vengono invece confrontati per lo stesso periodo le variazioni degli indici con il tempo.

Indichiamo ora con $\left(x_{1}, y_{1}\right), \ldots,\left(x_{r}, y_{r}\right), \ldots,\left(x_{n}, y_{n}\right)$ le 38 coppie di valori che due indici assumono nei 38 anni studiati. Siano:

1) $X_{i}$ ed $Y_{i}$ i valori "veri " di $x_{i}$ ed $y_{i}\left({ }^{9},{ }^{10}\right)$;

2) $\bar{x}=\sum x_{i} / n$;

3) $\bar{y}=\sum y_{i} / n$; 
4) $\sigma_{x}^{2}, \sigma_{y}^{2}, \sigma_{x y}$ rispettivamente la varianza delle $x_{i}$, delle $y_{i}$ e la covarianza delle coppie $x_{i}, y_{i}$ :

$$
\begin{aligned}
\sigma_{x} & =\sqrt{\frac{1}{n} \sum\left(x_{i}-\bar{x}\right)^{2}} \\
\sigma_{y} & =\sqrt{\frac{1}{n} \sum\left(y_{i}-\bar{y}\right)^{2}} \\
\sigma_{x y} & =\frac{1}{n} \sum\left(x_{i}-\bar{x}\right) \quad\left(y_{i}-\bar{y}\right) .
\end{aligned}
$$

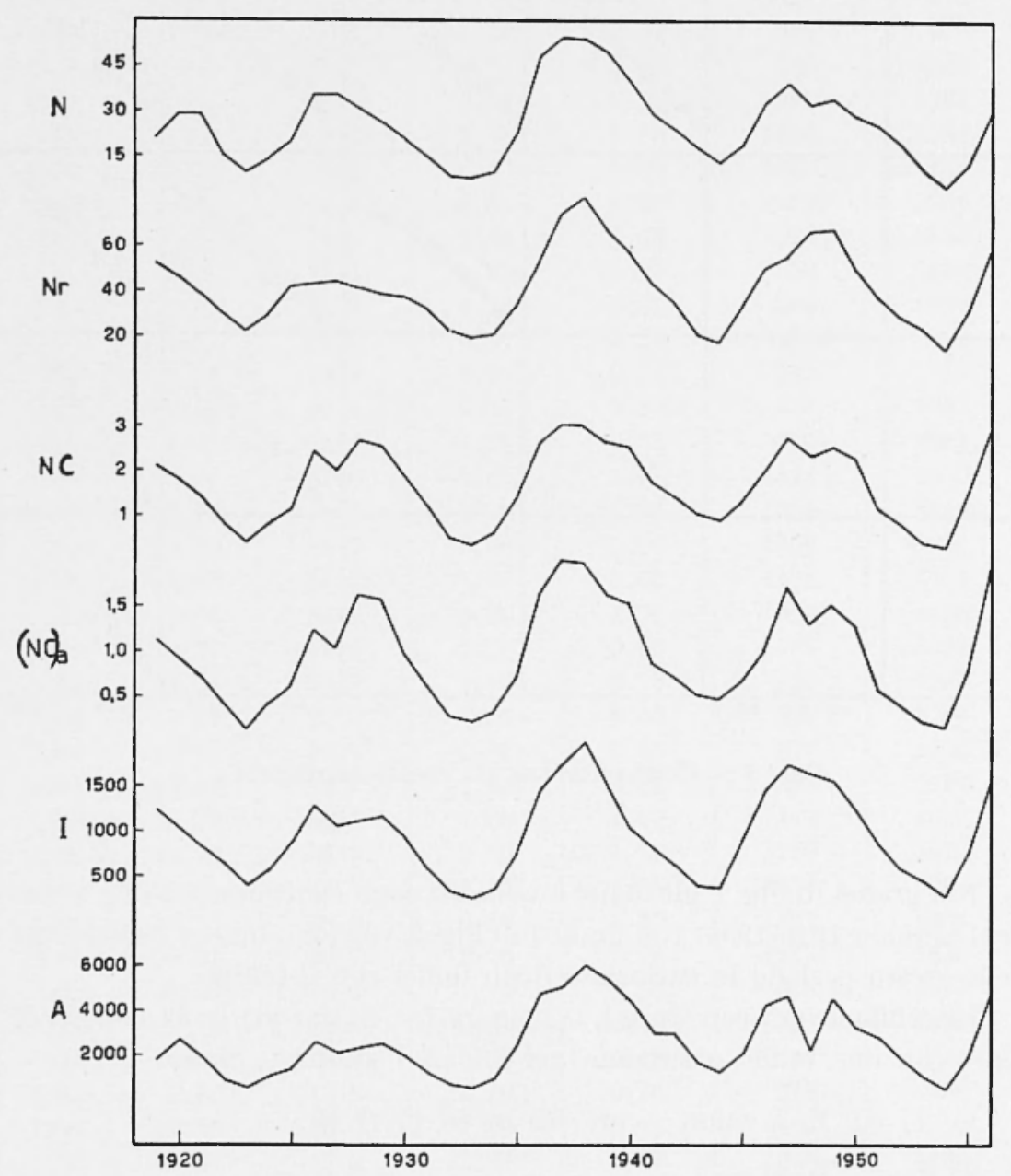

Fig. 2 - Variazioni col tempo degli indici analizzati. 
Definiamo "errori " $\delta_{i}$ e $\Delta_{i}$ le quantità

$$
\begin{aligned}
& \delta_{i}=x_{i}-X_{i} \\
& \Delta_{i}=y_{i}-Y_{i}\left({ }^{9},{ }^{10}\right) .
\end{aligned}
$$

Seguendo A. Wald $\left({ }^{8},{ }^{10}\right)$ supponiamo che:

1) gli errori $\delta_{i}$ abbiano distribuzioni identiche e non siano correlati;

2) gli errori $\Delta_{i}$ abbiano distribuzioni identiche e non siano correlati;

3) gli errori $\delta_{i}$ e $\Delta_{i}$ non siano correlati;

4) sia

$$
Y_{i}=\alpha X_{i}+\beta
$$

5) sia

$$
\left|\sum_{1}^{n / 2} x_{i}-\sum_{\frac{n}{2}+1}^{n} x_{i}\right| / n
$$

una quantità maggiore di zero.

Posto

$$
\begin{aligned}
& a_{1}=\left[\sum_{1}^{n / 2} x_{i}-\sum_{\frac{n}{2}+1}^{n} x_{i}\right] / n ; \\
& a_{2}=\left[\sum_{1}^{n} i / 2 y_{i}-\sum_{\frac{n}{2}+1}^{n} y_{i}\right] / n ; \\
& a-\frac{a_{2}}{a_{1}} ; \\
& b=\bar{y}-a \bar{x} ;
\end{aligned}
$$

si ha, per la legge dei grandi numeri $\left({ }^{9},{ }^{10}\right)$,

$$
\begin{aligned}
& \alpha \approx a \\
& \beta \approx b
\end{aligned}
$$

Indicando con $\sigma_{s}^{2}$ e $\sigma_{A}^{2}$ le varianze dei $\delta_{i}$ e $\Delta_{i}$ si ha, sempre per la legge dei grandi numeri $\left({ }^{9},{ }^{10}\right)$,

$$
\begin{aligned}
\sigma_{\delta} \approx \sqrt{\frac{n}{n-1}\left(\sigma_{x}^{2}-\frac{\sigma_{x v}}{a}\right)} \\
\sigma_{\Delta} \approx \sqrt{\frac{n}{n-1}\left(1 \sigma_{y}^{2}-a \sigma_{x y}\right)}
\end{aligned}
$$

Indicando infine con $r$ il coefficiente di correlazione fra gli $x_{\boldsymbol{t}}$ e gli $y_{\boldsymbol{i}}$ si ha

$$
r=\frac{\sigma_{x y}}{\sigma_{x} \sigma_{y}}
$$


Tabella VI. - Matrice dei Coefficienti di CoRrelazione.

\begin{tabular}{|c|c|c|c|c|c|c|c|}
\hline$y \quad x$ & $N$ & $N_{r}$ & $N . C$. & $(N . C .)_{a}$ & $I$ & $\therefore$ & $\Sigma$ \\
\hline$N$ & & 0.90 & 0.88 & 0.88 & 0.88 & 0.93 & 4.47 \\
\hline$N_{r}$ & 0.90 & & 0.89 & 0.89 & 0.95 & 0.90 & 4.53 \\
\hline N.C. & 0.88 & 0.89 & & 0.98 & 0.93 & 0.86 & 4.54 \\
\hline$(N . C .)_{a}$ & 0.88 & 0.89 & 0.98 & & 0.92 & 0.88 & 4.55 \\
\hline$I$ & 0.88 & 0.95 & 0.93 & 0.92 & & 0.89 & 4.57 \\
\hline$A$ & 0.93 & 0.90 & 0.86 & 0.88 & 0.89 & & 4.46 \\
\hline$\Sigma$ & 4.47 & 4.53 & 4.54 & 4.55 & 4.57 & 4.46 & \\
\hline
\end{tabular}

Tabella VII. - Matrice DELle Radici QUADRate delle VARIANze DEgLI " ERRORI " $\delta$, DELLE $x$.

\begin{tabular}{|c|c|c|c|c|c|c|}
\hline$y \quad x$ & $N$ & $N_{r}$ & $N . C$. & $(N . C .)_{a}$ & $I$ & il \\
\hline$N$ & & 3.1 & $i$ & $i$ & $i$ & 120 \\
\hline$N_{r}$ & 1.9 & & 0.026 & 0.11 & $i$ & $i$ \\
\hline$N . C$. & 4.2 & 3.7 & & 0.13 & 150 & 420 \\
\hline$(N . C .)_{a}$ & 3.7 & 1.8 & $i$ & & 130 & 350 \\
\hline$I$ & 3.7 & 3.1 & 0.059 & 0.12 & & 120 \\
\hline$A$ & 1.5 & $i$ & $i$ & $i$ & $i$ & \\
\hline$\Sigma \sigma_{3}$ & 15.0 & 11.7 & 0.085 & 0.36 & 280 & 1010 \\
\hline$\vec{\sigma}$ & 3.0 & 2.9 & 0.042 & 0.12 & 140 & 250 \\
\hline $\bar{x}$ & 25 & 41 & 1.6 & 0.92 & 950 & 2600 \\
\hline $100 \frac{\overline{\sigma_{1}}}{x}$ & $12 \%$ & $7 \%$ & $3 \%$ & $13 \%$ & $15 \%$ & $10 \%$ \\
\hline
\end{tabular}




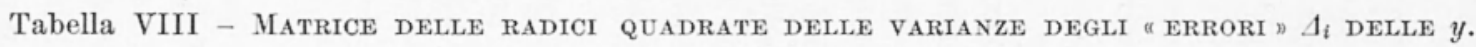

\begin{tabular}{|c|c|c|c|c|c|c|c|c|c|c|}
\hline$y$ & $N$ & $N_{r}$ & N.C. & $(N . O \cdot)_{a}$ & $I$ & A & $\Sigma \sigma_{\Delta}$ & $\bar{\sigma}_{\Delta}$ & $\bar{y}$ & $100 \frac{\mathrm{y}_{\bar{\sigma} \Delta}^{-}}{y}$ \\
\hline$N$ & & 5.1 & 6.6 & 6.1 & 6.5 & 4.7 & 29.0 & 5.8 & 25 & $23 \%$ \\
\hline$N_{r}$ & 6.9 & & 7.7 & 7.1 & 6.4 & 7.7 & 35.8 & 7.2 & 41 & $18 \%$ \\
\hline N.O. & 0.32 & 0.35 & & $i$ & 0.18 & 0.37 & 1.22 & 0.31 & 1.6 & $19 \%$ \\
\hline$(N . C .)_{a}$ & 0.22 & 0.25 & 0.13 & & 0.16 & 0.24 & 1.00 & 0.20 & 0.92 & $22 \%$ \\
\hline$I$ & 190 & 120 & 170 & 150 & & 210 & 840 & 170 & 950 & $18 \%$ \\
\hline$A$ & 530 & 670 & 820 & 760 & 770 & & 3350 & 710 & 2600 & $27 \%$ \\
\hline
\end{tabular}


La ricerca degli indici più adatti per la descrizione dell'attività dei filamenti solari è stata eseguita applicando due metodi statistici diversi.

Il primo metodo è consistito nel calcolo dei coefficienti di correlazione $r$ di ciascun indice rispetto a tutti gli altri. Diremo un indice $I_{1}$ migliore (peggiore) di un indice $I_{2}$ quando la somma dei coefficienti di correlazione fra l'indice $I_{1}$ e gli altri indici è maggiore (minore) della somma dei coefficienti di correlazione fra l'indice $I_{2}$ e gli altri indici.

Il secondo metodo si è basato invece sull'analisi dei $\sigma_{\delta}$ e $\sigma_{\Delta}$. Naturalmente $\sigma_{\delta}$ non dipende dall'indice $y$ considerato; analogamente $\sigma_{\Delta}$ non dipende dall'indice $x$ considerato. Si potrebbe quindi dire che un indice $I_{1}$ è migliore (peggiore) di un indice $I_{2}$ quando le quantità $\sigma_{\delta} / \bar{x}=\sigma_{\Delta} / y$ relative all'indice $I_{-}$siano minori (maggiori) delle quantità $\sigma_{\delta} / \bar{x}=\sigma_{A} / \bar{y}$ relative all'indice $I_{.}$. Quando però si assumano come valori dei $\sigma_{\delta}$ e $\sigma_{A}$ i valori dei secondi membri delle [3.3] e [3.4] introducendo una approssimazione tanto più grossolana quanto più piccolo è $n$ (ricordiamo che, nel nostro caso, $n=38$ ), i $\sigma_{\delta}$ potranno dipendere anche dall'indice $y$ considerato ed i $\sigma_{A}$ potranno dipendere dall'indice $x$ considerato. Si dirà allora che un indice $I_{1}$ è migliore (peggiore) di un indice $I_{2}$ quando la somma delle quantità $\sigma_{\delta} / \bar{x}$ dell'indice $I_{1}$ calcolate rispetto a tutti gli altri indici è minore (maggiore) della somma delle $\sigma_{\delta} / x$ dell'indice $I_{2}$ calcolate rispetto a tutti gli altri indici. Anche la somma delle quantità $\sigma_{\Delta} / \bar{y}$ dell'indice $I_{1}$ calcolate rispetto a tutti gli altri indici dovrà essere minore (maggiore) della somma delle quantità $\sigma_{\Delta} / y$ dell'indice $I_{2}$ calcolate rispetto a tutti gli altri indici.

Nella Tabella VI è riportata la matrice dei coefficienti di correlazione $r$. Gli elementi della matrice sono stati calcolati tutti per controllo. Dall'esame della matrice si deduce che, in prima approssimazione, i vari indici impiegati per la descrizione dell'attività dei filamenti solari sono in pratica equivalenti. L'indice migliore sembra essere l'indice rotazionale $I$ secondo Meudon e gli indici peggiori sembrano essere il numero giornaliero di fenomeni $N$ e l'area coperta giornalmente $A$.

Nelle Tabelle VII e VIII sono riportate le radici quadrate delle varianze rispettivamente degli " errori » $\delta_{i}$ delle $x$ e degli errori $\Delta_{i}$ delle $y$. Il fatto che alcuni valori siano immaginari può essere attribuito alla non completa validità delle ipotesi formulate. Nelle Tabelle VII ed VIII sono riportate anche le somme e le medie delle radici quadrate delle varianze degli errori; le medie degli indici considerati e le medie percentuali delle radici quadrate delle varianze degli errori. Il fatto che i valori dell'ultima riga della Tabella VII siano notevolmente diversi da quelli dell'ultima colonna di Tabella VIII, attribuibile anch'esso alla 
non completa validità delle ipotesi, impedisce l'applicazione del nostro secondo metodo.

La Fig. 1, in cui sono state riportate le rette definite dalla [3.1] e [3.2], e la Fig. 2 mostrano le buone correlazioni esistenti fra i diversi indiei.

Possiamo quindi concludere che tutti gli indici utilizzati per la descrizione dell'attività dei filamenti solari sembrano essere in pratica equivalenti anche se vi è qualche debole ragione per preferire l'indice rotazionale $I$ secondo Meudon e scartare il numero giornaliero di fenomeni $N$ e l'area coperta giornalmente $A$.

Firenze - Osservatorio Astrofisico di Arcetri, Dicembre 1964.

\section{BIBLIOGRAFIA}

(') K. O. Kinpenueuer, The Solar System. (Ed. Kuiper) 1 (1953).

C. DE JAGER, IIandb. Phys. (Ed. Flugge) 52 (1959).

R. G. Atilay and C. S. Warwick, Advane. Geophys. 9 (1962).

${ }^{(2)}$ Kodailianal Obs., Bulletin No 62 e segg.

( ${ }^{3}$ Paris-Meudon Obs., Ann. $6^{\circ}$ e successivamente Cartes Solaires.

(4) Character figures of solar phenomena e successivamente Quarterly Bulletin on Solar Activity, 1-5.

(5) G. Righini, G. Godoli, Sui numeri caratteristici dell'attività solare. "Ann. Geophys. ", 3, 501 (1950).

${ }^{\left({ }^{6}\right)}$ G. Godoli, Numeri caratteristici dei floceuli d'idrogeno e di calcio e dei filamenti d'idrogeno. "Ann. Geophys.", 7 (1954 e segg.).

(7) Osservatorio Astrofisico di Arcetri. Oss. e Mem. Fasc. 77 e segg.

${ }^{8}{ }^{8}$ G. Riginn e G. Godoli, Riduzione del materiale spetlroeliografico raccolto alla torre solare di Arcetri nel periodo 1932.1949. Men. SAIt., 21, 333 (1950).

$\left({ }^{9}\right)$ Y. V. Linnik, Method of least squares and principles of the theory of observations. Pergamon Press, 309 (1961).

${ }^{\left({ }^{10}\right)} \mathrm{A} . \mathrm{W}_{\mathrm{ALD}}$, The fitting of straight lines if both variables are subject to errors. "Ann. Math. Statistics", 11, 284 (1940). 